\title{
Mucineus cystadenoma of the appendix presenting as hydrosalpin $x$ - a pitfall in gynaecological medical imaging
}

\author{
Lenka Galajdova • Geert Braems • Kathleen Lambein • \\ Wim Ceelen • Rudy Van den Broecke
}

Received: 20 January 2008 / Accepted: 11 March 2008/Published online: 17 April 2008

(C) Springer-Verlag 2008

\begin{abstract}
Mucinous cystadenomas of the appendix represent a very small proportion of the appendiceal pathology, yet, they can lead to a life-threatening condition, such as pseudomyxoma peritonei (PMP), if the diagnosis is delayed. Due to the tubular shape and the proximity of the right adnex, the misdiagnosis of a hydrosalpinx, a condition much more common in women and not requiring an immediate intervention, could be made. We describe a case of a 19-year-old girl presenting with symptoms of acute low abdominal pain. According to the medical imaging (ultrasound and magnetic resonance), a torsion of known chronic hydrosalpinx was suspected and an urgent laparoscopy was performed revealing, surprisingly, an enlarged appendix. Laparoscopic appendectomy was performed without difficulty. The pathology report identified a mucinous cystadenoma. Despite the recent evolution of medical imaging techniques, a misdiagnosis of the nature of a pelvic mass is still possible, leading, occasionally, to the delay of an appropriate treatment. In any case of doubt, a diagnostic laparoscopy should be performed as a golden standard in the diagnosis and management of pelvic adnexal masses in women.
\end{abstract}

L. Galajdova $(\bowtie) \cdot$ G. Braems $\cdot$ R. Van den Broecke

Women's Health Department, University Hospital Ghent,

De Pintelaan 185,

9000 Ghent, Belgium

e-mail: lenka.galajdova@ugent.be

K. Lambein

Pathology Department, University Hospital Ghent,

Ghent, Belgium

W. Ceelen

General Surgery Department, University Hospital Ghent,

Ghent, Belgium
Keywords Appendix · Appendiceal pathology . Mucinous cystadenoma - Appendiceal tumour . Mucinous neoplasm of the appendix · Hydrosalpinx · Pelvic mass · Pseudomyxomatosis peritonei - Ovarian mass . Ovarian cyst $\cdot$ Medical imaging $\cdot$ Pelvic ultrasound

\section{Introduction}

A tubular-shaped structure found during ultrasound gynaecological examination is routinely considered as being a hydrosalpinx, a pathology treated mostly conservatively, except in the case of infertility or chronic pelvic pain. Appendiceal disorders, on the contrary, generally require an immediate intervention. Our case demonstrates a pitfall in medical imaging, including magnetic resonance imaging (MRI), in the differentiation between those two conditions and stresses the importance of diagnostic laparoscopy in doubtful cases to avoid an unacceptable delay in the treatment of potentially life-threatening illnesses.

\section{Case}

A 19-year-old white nulligravida using oral contraception was admitted with acute right iliac fossa pain, nausea and vomiting. Clinical examination revealed abdominal tenderness and rebound pain in the right lower abdominal quadrant and around the umbilicus. Vaginal bimanual palpation and mobilisation of the uterus was rather painful. The patient was pale and in the foetus position, and her temperature was normal. Biochemical inflammatory markers (C-reactive protein $0.2 \mathrm{mg} / \mathrm{dl}$, white blood cells [WBC] $5.710 \mathrm{E} 3 / \mu \mathrm{l})$ and human chorionic gonadotropin (hCG) $(<5 \mathrm{mU} / \mathrm{ml})$ were negative. Vaginal ultrasonography 


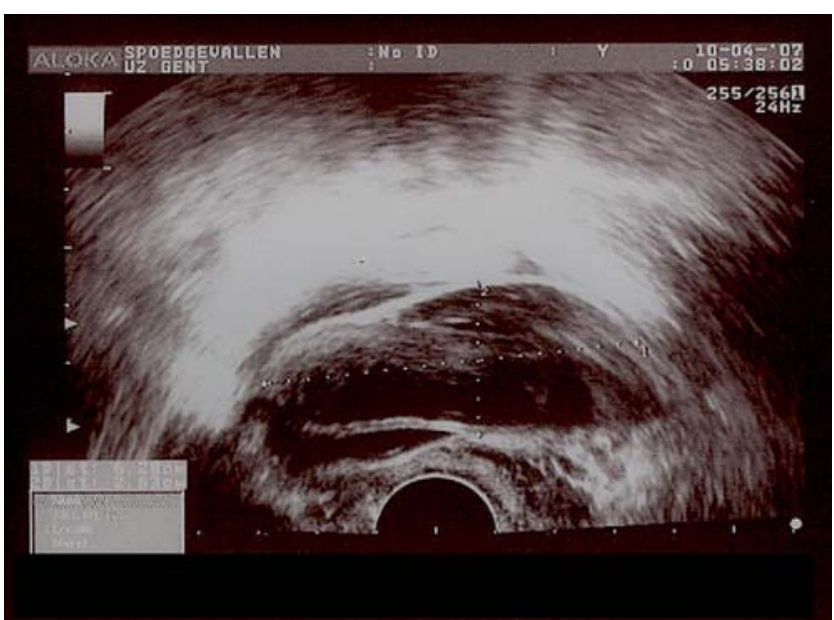

Fig. 1 Echography of the tubular structure

showed a tubular structure with dimensions $3 \times 7 \mathrm{~cm}$ in the Douglas pouch suggestive of a hydrosalpinx (Fig. 1).

This was her fourth similar episode within a year. The symptoms had always subsided after conservative treatment. The patient was followed up in the outpatient clinic for hydrosalpinx with episodes of intermittent pain attacks and she was put on the waiting list for laparoscopic salpingostomy/salpingectomy. This diagnosis was supported by findings of a sausage-shaped tubular structure in the Douglas pouch on MRI (Fig. 2).

In the view of the severity of the current episode and the suspicion of complete torsion, an emergency laparoscopy was performed. During the procedure, surprisingly, a complete normal internal genital status was found. In the Douglas pouch, a $6.5 \times 1.6-\mathrm{cm}$ large thin-walled appendix with clear mucinous content and signs of torsion was seen (Fig. 3).

An appendectomy was performed and the pathology report confirmed the diagnosis of mucinous cystadenoma of the appendix (Fig. 4).

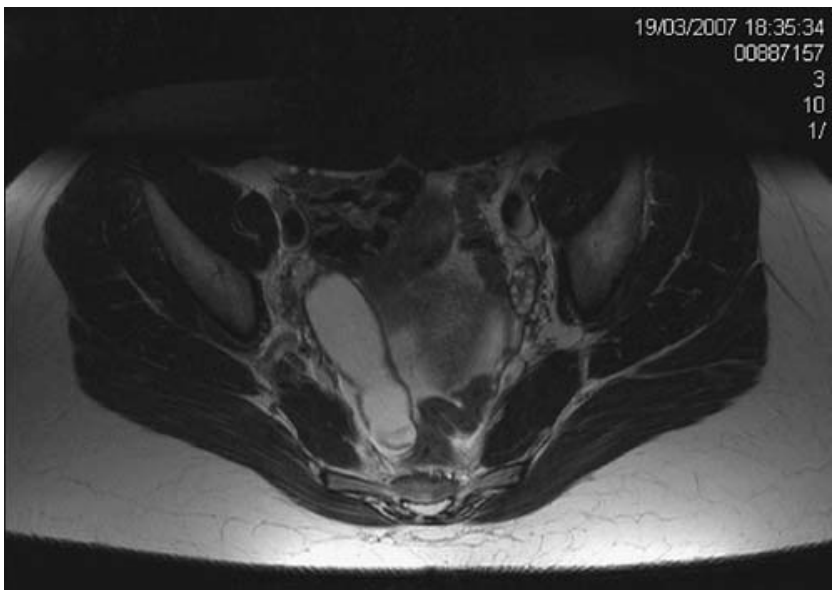

Fig. 2 Magnetic resonance imaging (MRI) of the tubular structure

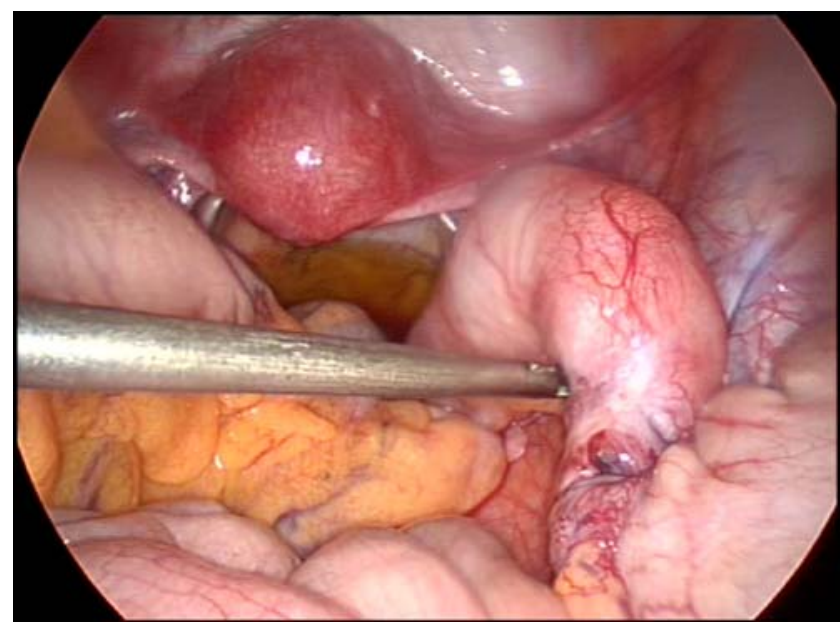

Fig. 3 View of the appendix during laparoscopy

The patient was discharged three days later in good general condition and is currently doing well.

\section{Comment}

Mucinous cystadenoma of the appendix belongs to the histological category of mucocoele (mucin-filled cystic dilatation) of the vermiform appendix [1] and is being found in $0.2-0.3 \%$ of all appendectomy specimens. Although rare, mucinous cystadenoma represents $63-84 \%$ of all mucocoeles [2]. These tumours are benign unless they disseminate through the wall of the appendix or rupture. A correlation between the intraperitoneal spread of neoplastic cells from these mucinous tumours and the clinical picture of pseudomyxoma peritonei (PMP) has been found, though clear evidence of a causal relationship is missing. PMP is a unique slowly progressing condition characterised by extensive mucus accumulation within the abdomen and

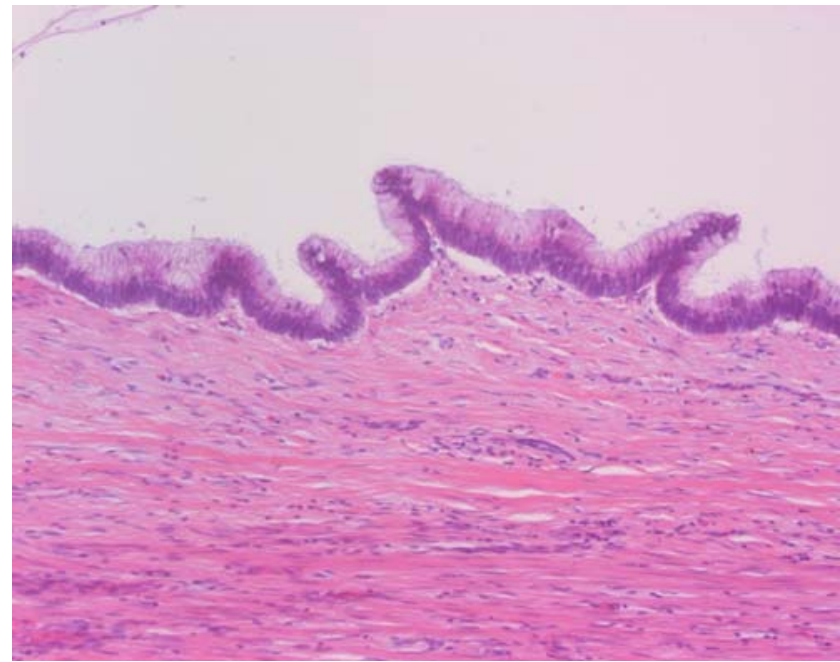

Fig. 4 Histopathology 


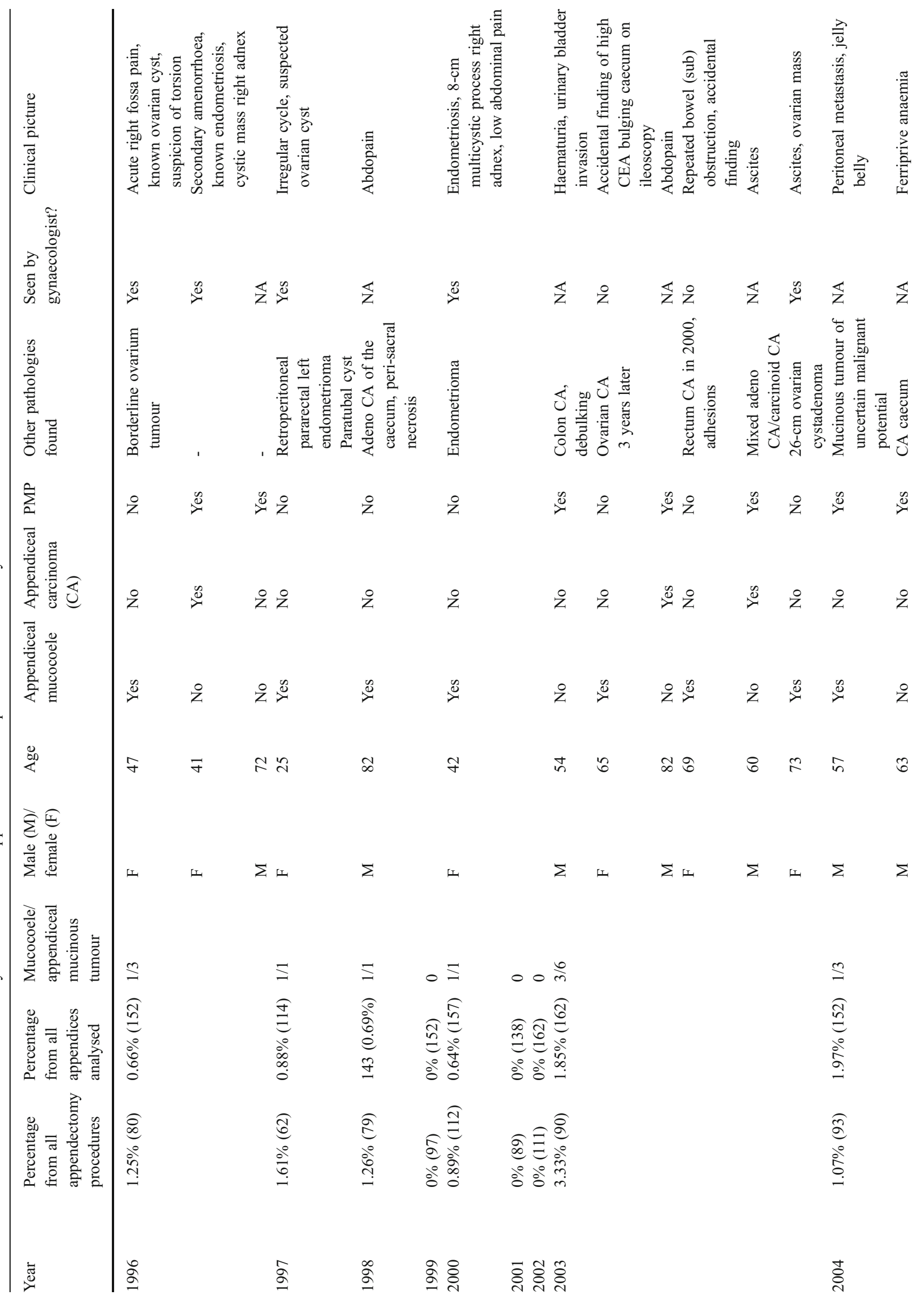




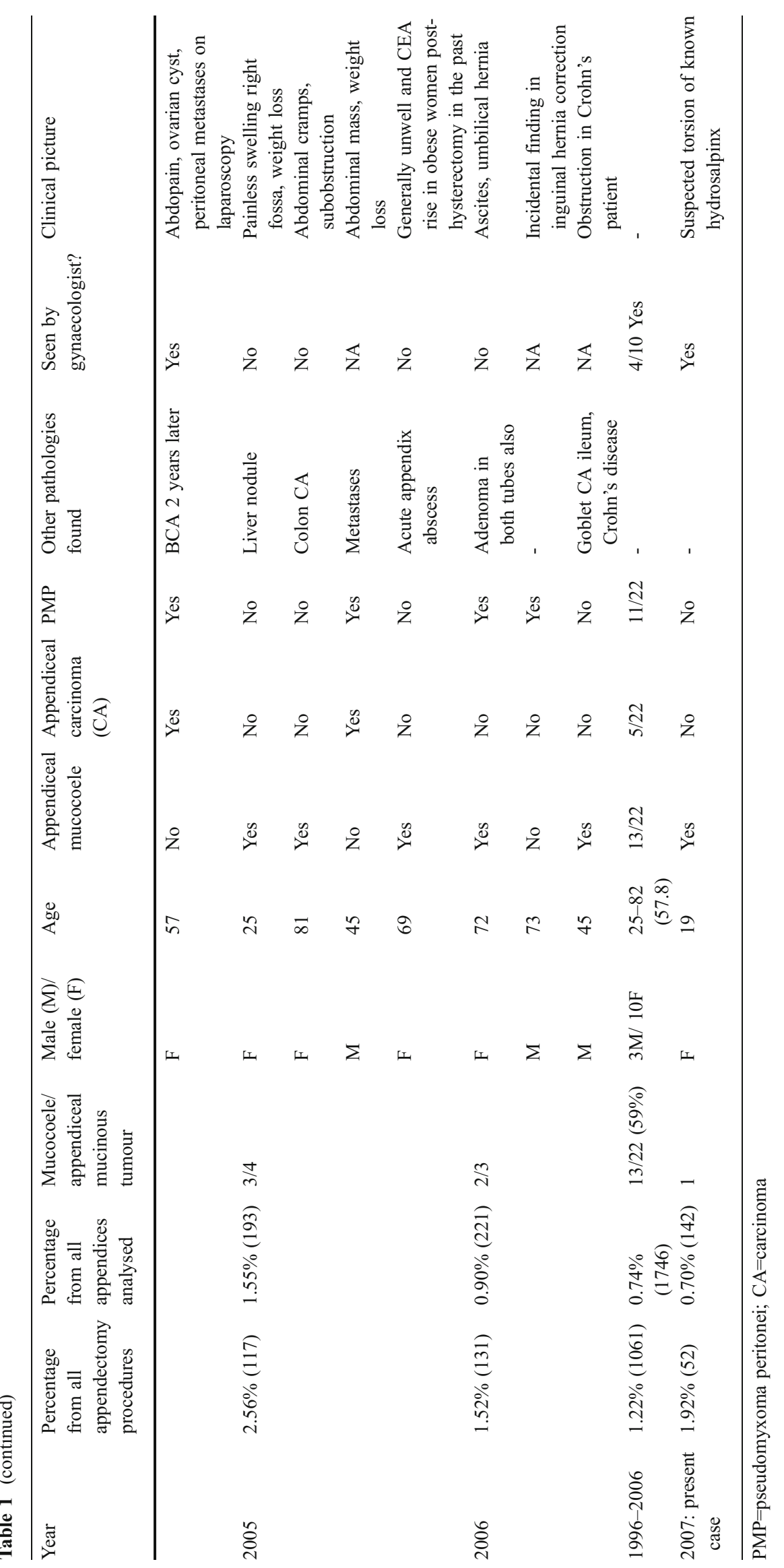


pelvis, gradually filling the peritoneal cavity, resulting in the characteristic "jelly belly" and leading, inevitably, to intestinal obstruction, nutritional compromise and death, unless definitively treated. Cytoreductive surgery and heated intraoperative intraperitoneal chemotherapy increase the survival rates from zero to approximately $80 \%[3,4]$.

PMP is more common in women between the ages of 40 to 60 years, with an incidence about 1 per million per year [5] and is found unexpectedly in 2 of 10,000 laparotomies [6]. Although it has been reported as originating from many intra-abdominal organs, in the majority of cases, an ovarian of appendiceal cystadenoma or cystadenocarcinoma has been implicated as the primary site.

An association between appendiceal mucocoeles and other tumours involving the gastrointestinal tract, ovary, breast and kidney has also been described $[5,6]$.

In the view of the rarity of this condition, an error in the diagnosis and treatment may easily occur [4].

Tubal damage is a cause of infertility in about $25 \%$ of infertile women. An infective process mediated mostly by chlamydia damages the intraluminal architecture and causes the generation of fluid-filled hydrosalpinges by an unknown mechanism [7].

Hydrosalpinges are usually tubular in shape and may have incomplete septations or nodules in its wall- "beadson-a-string" sign [8]. On the ultrasound, it can also mimic an ovarian cyst [7,9]. With the increasing use of transvaginal ultrasound scanning by gynaecologists, it is important to be aware of this. Unfortunately, colour Doppler energy (CDE) imaging and the evaluation of CA 125 plasma concentrations do not seem to increase the accuracy of Bmode transvaginal ultrasonography in differentiating hydrosalpinx from other adnexal masses $[8,10,11]$.

As our case shows, even the MRI can be misleading in establishing the correct preoperative diagnosis.

The young age of the patient in our case and pain originating around the umbilicus were more typical for an appendiceal pathology.

Without acute deterioration of the condition requiring an urgent intervention, the delay of the correct management could have occurred until rupture of the appendix with dissemination of the mucinous cells in the peritoneal cavity, calling for chemotherapy in an adolescent.

Appendiceal tumours, though uncommon, should be included in the differential diagnosis of pelvic masses and acute lower abdominal pain in women, especially in the absence of inflammatory markers. Despite the recent evolution of imaging techniques, they are not pitfall-free and diagnostic laparoscopy remains the reference standard in the diagnosis and treatment of female pelvic pathology, thus, "in dubio non abstine."

Additionally, analysing our own hospital data, we found, surprisingly, that, in a university hospital setting, the prevalence of appendiceal tumours could be much higher than expected (Table 1). In 2006, there were 131 appendectomies performed and 221 appendices sent for anatomopathologic analysis (including debulkings for ovarian carcinoma or colon resections). Of those, there were two mucineus cystadenomas $(1.52 \%$ or $0.90 \%$, respectively) identified, one of them as a part of PMP $(0.76 \%$ and $0.45 \%$, respectively) and five adenocarcinomas (3.81\% and $2.26 \%$, respectively, all of them being metastatic lesions of ovarium, colon or stomach adenocarcinomas). This strongly contrasts with the literature findings reporting appendiceal mucinous tumours in $0.1-0.3 \%$ of all appendectomy specimens, leaving us with a prevalence ten times higher.

\section{References}

1. Misdraji J, Yantiss RK, Graeme-Cook FM, Balis UJ, Young RH (2003) Appendiceal mucinous neoplasms: a clinicopathologic analysis of 107 cases. Am J Surg Pathol 27(8):1089-1103

2. Alrefaie W, Katz MH, Easter DW, Yi SE, Weidner N, Savides TJ, Moossa AR, Bouvet M (2004) Simultaneous serous cystadenoma of the pancreas and mucinous cystadenoma of the appendix. JOP 5(2):97-100

3. Pseudomyxoma Peritonei Specialty Service. Sugarbaker Oncology Associates (Specialty Section for the Treatment of Appendix Cancer) and the Pseudomyxoma Peritonei Syndrome. Available online at: http://www.surgicaloncology.com/pmp.htm

4. Atlas of Appendix Cancer andPseudomyxoma Peritonei. Available online at: http://www.surgicaloncology.com/attitle.htm

5. Bocca SM, Perry R, Oehninger S (2005) Pseudomyxoma peritonei: a rare differential diagnosis of hydrosalpinx. Fertil Steril 84(4):1017

6. Cancer of the appendix and pseudomyxoma peritonei. Available online at: $\mathrm{http}: / /$ www.uptodate.com/patients/content/topic.do?topicKey= gicancer $/ 8601$

7. Griffiths AN, Bhal PS, D'Angelo A (2003) An atypical hydrosalpinx: a differential diagnosis of an ovarian cyst. J Obstet Gynaecol 23(4):452-453

8. Guerriero S, Ajossa S, Lai MP, Mais V, Paoletti AM, Melis GB (2000) Transvaginal ultrasonography associated with colour Doppler energy in the diagnosis of hydrosalpinx. Hum Reprod 15(7):1568-1572

9. Shukla R (2004) Isolated torsion of the hydrosalpinx: a rare presentation. Br J Radiol 77(921):784-786

10. Sonographic differentiation of benign versus malignant adnexal masses. Available online at: http://www.uptodate.com/patients/ content/topic.do?topicKey=gen_gyne/14319

11. Ohara N, Narita F, Murao S (2003) Isolated torsion of haematosalpinx associated with tubal endometriosis. J Obstet Gynaecol 23 (4):453-454 